\title{
Risk factors predicting the occurrence of metachronous ovarian metastasis of gastric cancer
}

\author{
Jianpeng Gao ${ }^{1,2 \#} \wedge$, Weiwei Weng ${ }^{2,3 \#}$, Xiaofei $\mathrm{Qu}^{2,4 \#}$, Binhao Huang ${ }^{1}$, Yu Zhang $^{5}$, Zhenglun Zhu $^{6}$ \\ ${ }^{1}$ Department of Gastric Surgery, Fudan University Shanghai Cancer Center, Shanghai, China; ${ }^{2}$ Department of Oncology, Shanghai Medical College, \\ Fudan University, Shanghai, China; ${ }^{3}$ Department of Pathology, Fudan University Shanghai Cancer Center, Shanghai, China; ${ }^{4}$ Cancer Institute, \\ Fudan University Shanghai Cancer Center, Shanghai, China; ${ }^{5}$ Department of Pathology, Ruijin Hospital Affiliated to Shanghai Jiaotong University \\ School of Medicine, Shanghai, China; ${ }^{6}$ Department of General Surgery, Shanghai Key Laboratory of Gastric Neoplasms, Shanghai Institute of \\ Digestive Surgery, Ruijin Hospital Affiliated to Shanghai Jiaotong University School of Medicine, Shanghai, China \\ Contributions: (I) Conception and design: J Gao, Z Zhu; (II) Administrative support: J Gao, Z Zhu; (III) Provision of study materials or patients: J \\ Gao, W Weng, Z Zhu; (IV) Collection and assembly of data: J Gao, W Weng, X Qu, B Huang, Y Zhang; (V) Data analysis and interpretation: J Gao, \\ W Weng, X Qu, Y Zhang, Z Zhu; (VI) Manuscript writing: All authors; (VII) Final approval of manuscript: All authors. \\ "These authors contributed equally to this work. \\ Correspondence to: Jianpeng Gao. Department of Gastric Surgery, Fudan University Shanghai Cancer Center, No. 270 Dongan Road, Xuhui District, \\ Shanghai 200032, China. Email: jianpeng.gao@shca.org.cn. Zhenglun Zhu. Ruijin Hospital Affiliated to Shanghai Jiaotong University School of \\ Medicine, No. 197 Ruijin 2nd Road, Huangpu District, Shanghai 200025, China. Email: zzl11703@rjh.com.cn.
}

Background: Ovarian metastasis following radical gastrectomy, also known as metachronous ovarian metastasis (MOM), pose a significant threat to the long-term survival of female gastric cancer (GC) patients. However, a mechanism to identify and characterize operated patients at high risk of developing MOM remains unknown. This retrospective study aimed to identify risk factors for the occurrence of MOM based on the profiling of clinicopathological parameters and expression of sex hormone receptors (SHR) of operated GC patients with and without ovarian relapse.

Methods: The clinicopathological data of 1,055 female GC patients from two medical centers who underwent surgery between January 2011 and December 2015 were reviewed. A total of 378 patients with and without the occurrence of MOM met the eligibility criteria, including the availability of medical records, adequacy of lymph node dissection, completeness of clinicopathological data, sufficient follow-up time, and no administration of neoadjuvant chemotherapy were selected for further analysis. Expressions of estrogen receptor alpha $(\mathrm{ER} \alpha)$, estrogen receptor beta $(\mathrm{ER} \beta)$, and progesterone receptor $(\mathrm{PR})$ were detected by immunohistochemical staining on the surgical specimens of patients, and retrospective statistical analyses identified independent risk factors for the occurrence of MOM. A risk prediction model in the format of a polygenic hazard score (PHS) for the occurrence of MOM was established by introducing and modifying the previously validated polygenic risk score (PRS)/PHS.

Results: A Cox regression-based multivariate analysis identified premenopausal with an HR of 3.15 (95\% CI, 1.66-5.98), more advanced pathological T stage with an HR of 3.79 (95\% CI, 2.14-6.69), more advanced pathological N stage with an HR of 1.85 (95\% CI, 1.35-2.54), and negative expression of ER $\beta$ with an HR of 0.33 (95\% CI, 0.15-0.7) as independent risk factors for the occurrence of MOM $(\mathrm{P}<0.01)$. Accordingly, a PHS for the occurrence of MOM was established, with 1-, 2-, and 3-year ovarian relapse rates for the highrisk group estimated at $17.8 \%, 33.7 \%$, and $46.2 \%$, respectively.

Conclusions: Premenopausal status, depth of tumor invasion, number of positive lymph nodes, and negative expression of ER $\beta$ were independent factors for the occurrence of MOM. More frequent follow-up examinations are recommended to provide timely diagnosis and medical intervention.

^ ORCID: 0000-0002-4627-6278. 
Keywords: Gastric cancer (GC); metachronous ovarian metastasis (MOM); risk factor; estrogen receptor beta (ER $\beta$ )

Submitted Mar 24, 2021. Accepted for publication May 21, 2021.

doi: $10.21037 /$ atm-21-1419

View this article at: https://dx.doi.org/10.21037/atm-21-1419

\section{Introduction}

Gastric cancer (GC) is one of the most common and deadly malignancies worldwide. Although extensive efforts are made to improve the long-term survival rate, it remains the $3^{\text {rd }}$ most lethal cancer, according to GLOBOCAN 2018 statistics (1). Distant metastasis occurs in approximately half of GC patients and significantly contributes to treatment failure and poor prognosis (2), and the ovary is a major target organ for the metastasis of multiple nongynecologic malignancies, especially gastric and colorectal cancer $(3,4)$. While ovarian metastasis may be synchronously identified at the initial diagnosis of GC, a proportion of patients develop ovarian relapse after radical gastrectomy, which is termed as metachronous ovarian metastasis (MOM) (5). MOM occurs in $0.3-6.7 \%$ of GC patients undergoing radical surgery, and its incidence rate reaches up to $41 \%$ by autopsy (6). The prognosis of GC patients with MOM is reportedly poor, as its median survival time is less than 14 months and 3-year survival rate less than $10 \%$ (7). Although previous studies have characterized the clinical features of these patients and discussed potentially beneficial treatment strategies, risk factors for the occurrence of MOM after initial surgical procedures remain largely unknown. One study published approximately 20 years ago reported a significant association between the number of positive lymph nodes and patient age on the risk of post-gastrectomy ovarian relapse (8). Correlation between the expression status of sex hormone receptors (SHR) such as estrogen receptor alpha (ER $\alpha$ ), estrogen receptor beta (ER $\beta)$, and progesterone receptor $(\mathrm{PR})$ and GC progression and its ovarian metastasis have also been investigated (9-11).

Nevertheless, the predictive role of SHR expression in the recurrent ovarian metastasis of operated GC patients remains unclarified. Therefore, we performed this retrospective study to characterize the independent risk factors for the occurrence of MOM based on the systematic profiling of clinicopathological parameters and the expression status of $\mathrm{ER} \alpha, \operatorname{Er} \beta$, and $\mathrm{PR}$ in operated GC patients. Accordingly, we further established a risk prediction model for MOM development to identify operated GC patients at high risk of ovarian relapse so that tailored follow-up examination and treatment could be applied promptly. We present the following article in accordance with the REMARK reporting checklist (available at http://dx.doi.org/10.21037/atm-21-1419).

\section{Methods}

\section{Patients}

We initially evaluated the medical records of 1,055 female GC patients receiving D2 gastrectomy in the Fudan University Shanghai Cancer Center and Ruijin Hospital affiliated to the Shanghai Jiaotong University School of Medicine from January 2011 to December 2015. The eligibility criteria for the study included the availability of medical records, adequacy of lymph node dissection, completeness of clinicopathological data, sufficient follow-up time, and no administration of neoadjuvant chemotherapy, which led to a total of 378 patients meeting all criteria and selected for further analysis (Figure 1). Clinicopathological data including age, the status of menstruation, vessel and lymphatic thrombus, WHO pathological classification, tumor differentiation, depth of tumor invasion (pathological T stage, $\mathrm{pT}$ stage in short), number of positive lymph nodes (pathological $\mathrm{N}$ stage, $\mathrm{pN}$ stage in short), and the American Joint Committee on Cancer (AJCC) staging system were collected and are summarized in Table 1. Among all reviewed patients, 55 developed ovarian relapse after receiving radical D2 gastrectomy followed by chemotherapy, mainly comprised of fluorouracil, oxaliplatin, calcium folinate, and docetaxel. Most patients with ovarian metastasis recurrence (90.9\%, 50/55) underwent exploratory laparotomy followed by either curative or palliative metastasectomy based on the distribution of metastatic foci remaining five patients did not undergo surgery and received palliative chemotherapy.

\section{Follow-up}

Follow-up was performed during outpatient visits at 


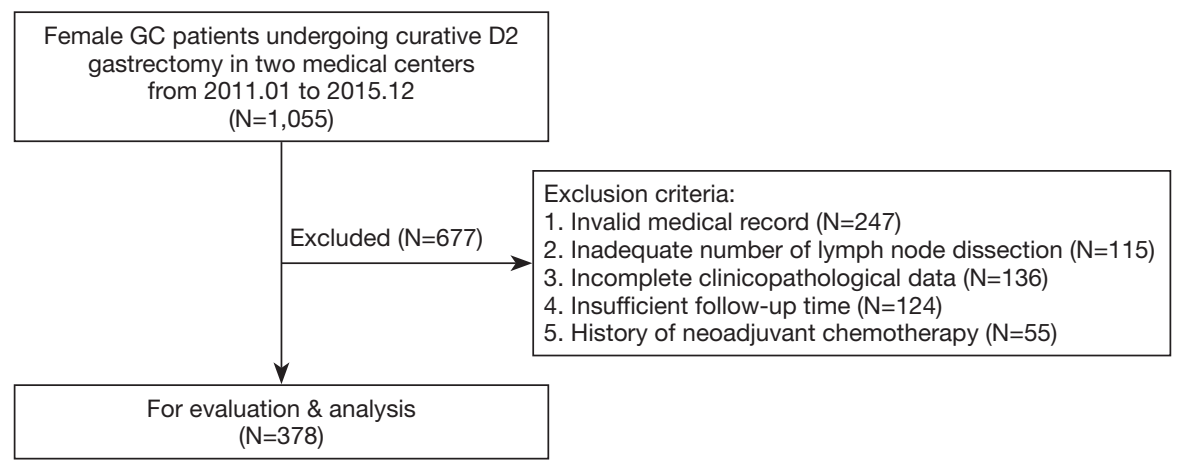

Figure 1 Schematic diagram of eligibility/exclusion criteria.

1 month after the operation and then at 3-6 months intervals in the first year and twice per year after that. Regular examinations included physical examination, tumor marker testing, and abdominal and pelvic ultrasonography. Abdominal and pelvic computed tomography (CT) with or without intravenous contrast was either performed every 6 months or when other regular examinations suggested pelvic occupation, and other auxiliary examinations such as positron emission tomography-computed tomography (PET-CT) and single-photon emission computed tomography (SPECT) were applied when distant metastasis was suspected. Confirmation of MOM was based on either pathological inspection of surgically removed metastatic foci or imaging diagnosis, including pelvic CT/PETCT scanning. For all 55 patients with the occurrence of MOM, metastatic foci-related information, including the laterality of ovarian foci, the presence/absence of ascites, distribution of metastatic foci, and tumor marker tests $3-$ 6 months before the diagnosis of MOM were collected and are summarized in Table 2. Information on the status and outcome of patients were obtained via admission records, outpatient clinic, follow-up telephone call, and E-mail. Follow-up was terminated when MOM was clinically and pathologically diagnosed, and the median follow-up time was 41 months.

\section{Pathology and immunobistochemistry}

Upon completion of the initial curative gastrectomy and secondary metastasectomy, fresh tumor tissues were fixed in $10 \%$ neutralized formalin, embedded in paraffin, and processed for hematoxylin and eosin (H\&E) staining, which were further used for pathological diagnosis and staging. Paraffin blocks were stored at room temperature within two weeks and at 4 degrees $C$ for long-term use. The 8 th edition of The American Joint Committee on Cancer (AJCC) was applied to determine the pathological TNM stage of primary GC based on the depth of tumor invasion (pT stage), number of tumor-invaded lymph nodes ( $\mathrm{pN}$ stage), and absence/presence of distant tumor metastasis (pM stage). Ovarian metastasis of GC was diagnosed according to (I) the history of primary gastric adenocarcinoma; (II) the histological correspondence between primary tumor and ovarian metastasis; and (III) the representative morphology by histological inspection such as poorly cohesive/signetring cells $(12,13)$.

Fixed and embedded paraffin blocks were processed into tissue microarray (TMA) to determine the expression status of $E R \alpha, E R \beta$, and PR on primary tumor specimens, and the construction and storage of TMA and immunohistochemical staining of these markers were performed according to the methodology published by Ryu et al. (9). Briefly, the specimens underwent sequential processes, including deparaffinization by xylene, rehydration by graded ethanol, and heat-induced epitope retrieval. Sections were then subjected to incubation with $\mathrm{H}_{2} \mathrm{O}_{2}$, phosphate-buffered saline (PBS) washing, and blocking with $3 \%$ hydrogen peroxide followed by pre-immune goat serum. Following this, the sections were incubated with primary antibodies against $\mathrm{ER} \alpha, \operatorname{Er} \beta$, and $\mathrm{PR}$, followed by incubation with secondary biotinylated anti-mouse/rabbit antibodies. Processed sections were visualized after incubation with red chromogen at $40^{\circ} \mathrm{C}$, and further counterstained using the Mayer hematoxylin method. The products of primary antibodies included anti-ER $\alpha$ (ab37438, dilution 1:200; Abcam, Cambridge, UK), anti-ER $\beta$ (ab288, dilution 1:100; Abcam), and anti-PR (ab16661, dilution 1:100; Abcam). The definition of positivity was based on the scoring system 
Table 1 Clinicopathological parameters of 378 female GC patients with or without MOM

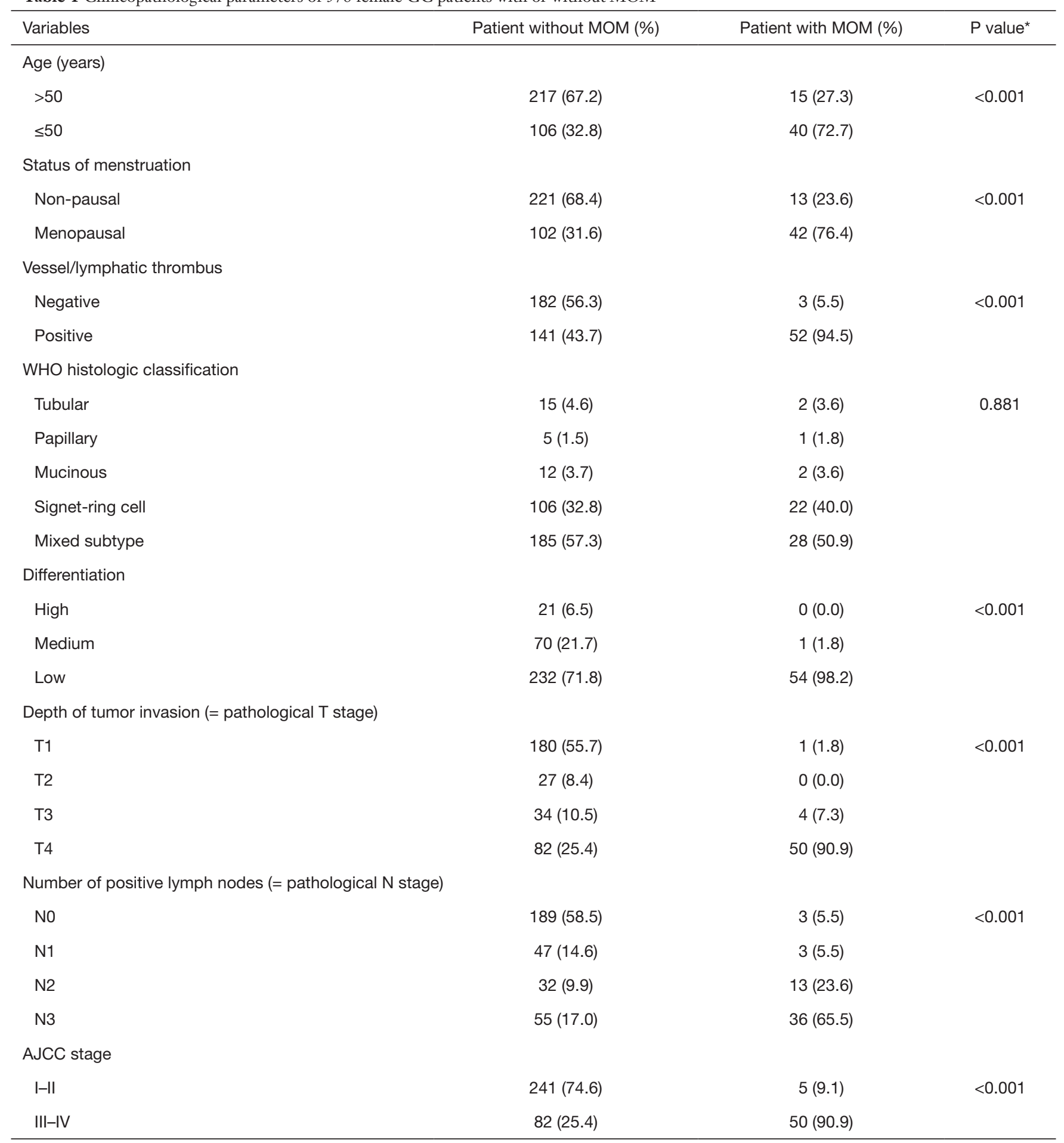

*, Chi-square test. GC, gastric cancer; WHO, World Health Organization; MOM, metachronous ovarian metastasis; AJCC, the American Joint Committee on Cancer. 
Table 2 Clinicopathological variables of 55 female GC patients with MOM

\begin{tabular}{|c|c|c|}
\hline Variables & Number & Percentage \\
\hline \multicolumn{3}{|l|}{ Laterality of ovarian metastasis } \\
\hline Unilateral & 14 & 25.5 \\
\hline Bilateral & 41 & 74.5 \\
\hline \multicolumn{3}{|l|}{ Ascites } \\
\hline Presence & 34 & 61.8 \\
\hline Absence & 17 & 30.9 \\
\hline Undetermined & 4 & 7.3 \\
\hline \multicolumn{3}{|l|}{ WHO pathological classification } \\
\hline Signet-ring cell & 19 & 40.4 \\
\hline Mixed subtype & 28 & 59.6 \\
\hline \multicolumn{3}{|l|}{ Distribution of metastatic foci } \\
\hline Confined to the ovary & 31 & 56.4 \\
\hline Disseminated in pelvic peritoneum & 7 & 12.7 \\
\hline $\begin{array}{l}\text { Disseminated in abdominal } \\
\text { peritoneum }\end{array}$ & 17 & 30.9 \\
\hline \multicolumn{3}{|l|}{ Tumor marker* } \\
\hline Normal & 13 & 35.1 \\
\hline Elevated CA125 & 3 & 8.1 \\
\hline Elevated CA199 & 2 & 5.4 \\
\hline Elevated CEA & 3 & 8.1 \\
\hline Elevated CA724 & 10 & 27.0 \\
\hline Elevated CA724 + CA199 & 3 & 8.1 \\
\hline Elevated CA199 + CA125 & 1 & 2.7 \\
\hline Elevated CA199 + CA242 & 2 & 5.4 \\
\hline
\end{tabular}

*, tumor markers were regularly tested for 37 patients with MOM. GC, gastric cancer; WHO, World Health Organization; MOM, metachronous ovarian metastasis.

introduced by Gan et al. (14), and the inspection and scoring were performed by two independent pathologists who were unaware of the clinical outcomes. The expression status of ER $\alpha, \operatorname{Er} \beta$, and PR are presented in Table 3.

\section{Statistical analysis}

Clinicopathological parameters, tumor marker test results, and the expression status of SHRs of surgical GC patients were included for statistical analyses. Spearman chi- squared test was used to compare the clinicopathological parameters and expression status of SHRs between surgical GC patients with and without MOM. The same statistical methodology was applied to compare tumor marker testing results between patients with ovarian relapse with confined and disseminated metastatic foci. Univariate and multivariate analyses were sequentially conducted using a Cox proportional hazards regression model to determine independent risk factors for the occurrence of MOM. As four independent risk factors, including menstrual status, depth of tumor invasion ( $\mathrm{pT}$ stage), the number of positive lymph node ( $\mathrm{pN}$ stage), and expression status of ER $\beta$ were determined, a polygenic hazard score (PHS) tool was introduced to establish the risk prediction model as previously described (15). The best cutoff value between high-risk and low-risk groups was defined and manifested via the Survminer R package (https://CRAN.R-project. $\mathrm{org} /$ package $=$ survminer). All statistical analyses were performed two-sided at a significance level of $\mathrm{P}=0.05$ with the application of $\mathrm{R}$ package version 3.5.1.

\section{Statement of ethics and consent}

The study was conducted in accordance with the Declaration of Helsinki (as revised in 2013). The study was approved by the local ethics committee of the Fudan University Shanghai Cancer Center (Number: 050432-41911D) and Ruijin hospital affiliated to Shanghai Jiaotong University School of Medicine [Number: (2018) Linlunshen No. 151]. Informed consent was obtained from all patients

\section{Results}

\section{Clinicopathological features of female GC patients with and without MOM}

Between January 2011 to December 2015, 1,055 female GC patients administered in the Fudan University Shanghai Cancer Center and Ruijin Hospital affiliated to Shanghai Jiaotong University School of Medicine for radical D2 gastrectomy were reviewed, and a total number of 378 patients meeting all eligibility criteria (described in the section of Methods) were further selected for this retrospective study (illustrated in Figure 1). The general clinicopathological parameters of all 378 patients are summarized in Table 1. The median follow-up period was 41 months (range, 3-93 months), and 55 patients developed MOM during the post-operative follow-up phase. 
Table 3 Expression status of SHRs of female GC patient with or without MOM

\begin{tabular}{lcc}
\hline Variables & Patients without the occurrence of MOM (\%) & Patients with the occurrence of MOM (\%) \\
\hline $\begin{array}{l}\text { ER } \alpha \text { expression } \\
\text { Negative }\end{array}$ & $297(92.0)$ & $51(92.7)$ \\
Positive & $26(8.0)$ & $4(7.3)$ \\
ER $\beta$ expression & & 1.000 \\
Negative & $181(56.0)$ & $47(85.5)$ \\
Positive & $142(44.0)$ & $8(14.5)$ \\
PR expression & & $41(74.5)$ \\
Negative & $227(70.3)$ & $14(25.5)$ \\
Positive & $96(29.7)$ & $<0.001$ \\
\hline
\end{tabular}

*, Chi-square test. SHR, sex hormone receptor; GC, gastric cancer; MOM, metachronous ovarian metastasis; ER $\alpha$, estrogen receptor $\alpha$; $E R \beta$, estrogen receptor $\beta$; PR, progesterone receptor.

Clinical features of the 55 patients with MOM were further characterized, revealing the interval between the initial diagnosis of GC and post-surgical ovarian relapse was on average 20 months (ranging from 3 to 72 months), with up to 40 patients developing MOM within the first two years of gastrectomy. Among all 55 patients, $72.7 \%$ (40/55) were under the age of $50,76.4 \%(42 / 55)$ were premenopausal, and the mean age at initial GC diagnosis was 43 years. Upon the discovery of a mass in the pelvic and/or lower abdominal cavity leading to the suspicion of ovarian relapse, $85.5 \%$ of patients (47/55) underwent secondary explorative laparotomy followed by either radical (R0) or palliative (R1) metastasectomy based on the distribution of metastatic foci. Of the 47 patients undergoing laparotomy, unilateral and bilateral ovarian metastasis were found in 13 and 34 patients, respectively. Pathological inspection of the primary gastric lesions showed that all were low differentiation adenocarcinoma with either signet-ring cell carcinoma (19/47) or mixed subtype (28/47) of histology, and the same findings were witnessed on their matching metastatic ovarian lesions, indicating the consistency of pathological types between paired primary and metastatic tumors. Remarkably, we also found that metastatic foci were either confined to the ovary in 25 operated patients and disseminated in the pelvic and/ or abdominal peritoneum in the other 22 . For the other eight patients receiving only palliative chemotherapy, radiographic/imaging examinations indicated that the ovarian tumors were unilateral/bilateral in $1 / 7$ cases and metastatic foci were confined/disseminated in $6 / 2$ cases, respectively. Additionally, the presence/absence of ascites was confirmed in 51 cases, with its presence in four cases was unclear due to technical limitations. All results are summarized in Table 2.

Serum tumor markers were routinely tested in 37 patients undergoing metastasectomy. Revision of the tumor markers tested 3-6 months before the diagnosis of MOM revealed that 13 patients $(35.1 \%)$ were negative for all markers, 3 (8.1\%) were positive for CA125, 2 (5.4\%) were positive for CA199, 3 (8.1\%) were positive for CEA, 10 (27.0\%) were positive for CA724, and 6 patients (16.2\%) were positive for multiple markers (summarized in Table 2). Notably, the positive rate of CA724 in patients with metastatic foci disseminated in the pelvic and/or abdominal peritoneum was borderline significantly higher than patients with metastatic foci confined to the ovary $(\mathrm{P}=0.05)$. This indicated that the metastatic foci of female GC patients with positive CA724 were prone to disseminate in the pelvic and/or abdominal cavity rather than remaining confined to the ovaries

\section{Expression status of SHRs of female GC patients with and without MOM}

As previous studies have indicated a significant association between the expression status of SHR and prognosis of patients both with and without ovarian metastasis $(9,11,14)$, we investigated whether SHR expression was correlated with MOM. Firstly, we profiled the expression status of SHRs including ER $\alpha, \operatorname{Er} \beta$, and PR on the primary 

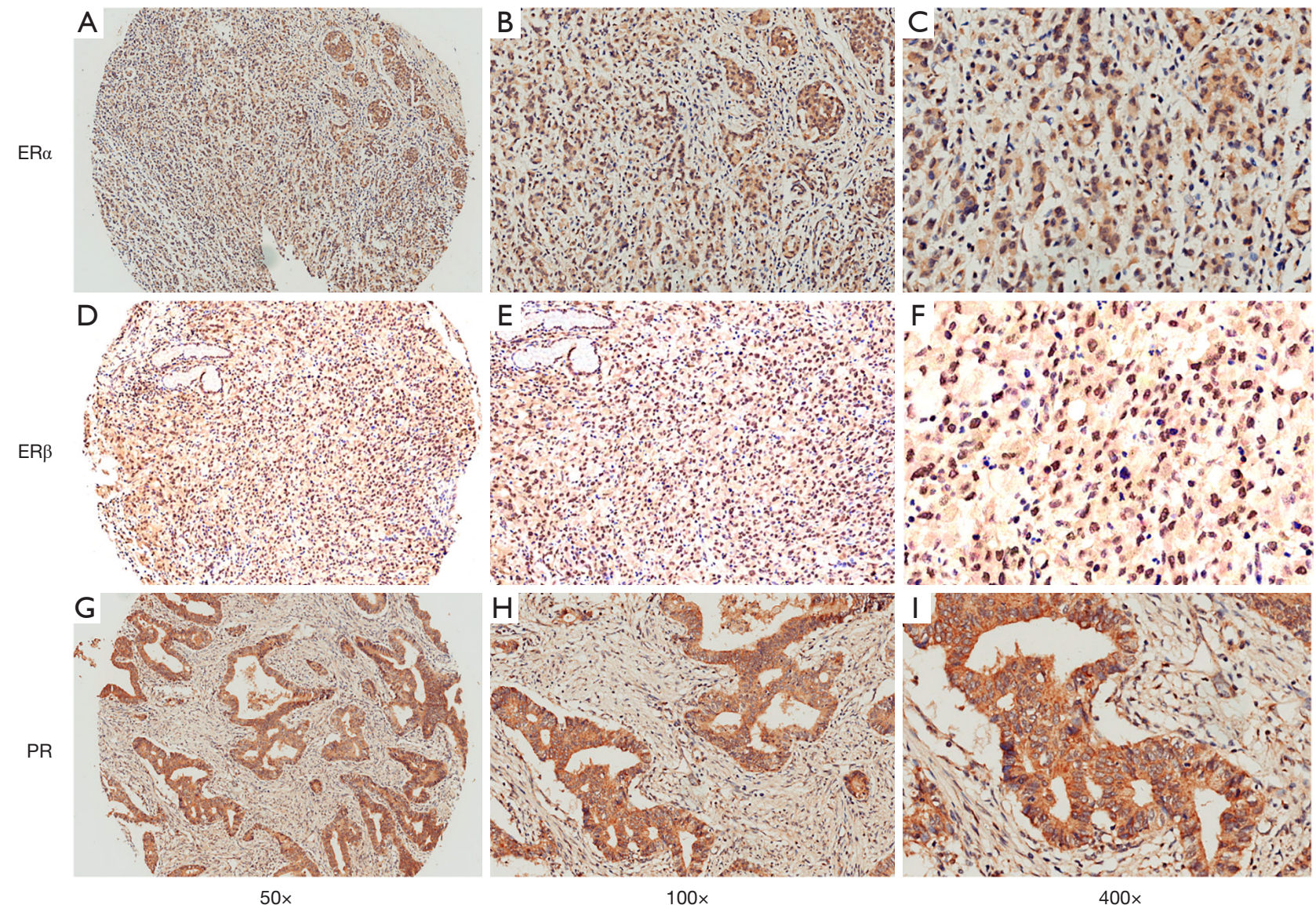

$100 x$

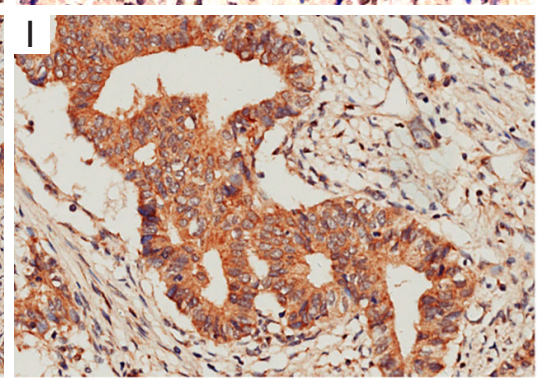

$400 x$

Figure 2 Representative image of immunohistochemical-based staining of ER $\alpha, E R \beta$, and PR in primary gastric cancer tissues. (A,B,C) Positive expression of ER $\alpha$ (A. $\times 50$; B. $\times 100$; C. $\times 400)$. (D,E,F) Positive expression of ER $\beta$ (D. $\times 50$; E. $\times 100$; F. $\times 400)$. (G,H,I) Positive expression of PR (G. $\times 50$; H. $\times 100$; I. $\times 400)$. ER $\alpha$, estrogen receptor $\alpha$; ER $\beta$, estrogen receptor $\beta$; PR, progesterone receptor.

GC specimens of all 378 patients by performing TMA -based immunohistochemical staining of these markers (demonstrated in Figure 2). A specific scoring system (described in Methods section) was applied to determine the positivity of $\mathrm{ER} \alpha / \mathrm{ER} \beta / \mathrm{PR}$ and showed that for the 323 patients without ovarian relapse after primary radical gastrectomy, the positive expression rate of $\mathrm{ER} \alpha, \mathrm{ER} \beta$, and PR was $8.0 \%, 44.0 \%$, and $29.7 \%$, respectively. However, in the 55 patients with MOM, the positive expression rate of $\mathrm{ER} \alpha, \mathrm{ER} \beta$, and $\mathrm{PR}$ was $7.3 \%, 14.5 \%$, and $25.5 \%$, respectively (summarized in Table 3), and the difference between the two groups was significant $(\mathrm{P}<0.001)$. On this basis, we further performed immunohistochemical staining of ER $\beta$ on the resected ovarian tumors of 47 GC-MOM patients undergoing secondary laparotomy and oophorectomy to evaluate the consistency of ER $\beta$ expression between paired primary and metastatic lesions.
This revealed that among eight ER $\beta(+)$ gastric tumors, seven had matching ovarian tumors with positive ER $\beta$ expression, but ER $\beta$ expression was not detected in the matching ovarian tumors of the other 39 ER $\beta$ (-) gastric tumors (illustrated in Figure S1).

\section{Identification of independent risk factors and establishment of a risk prediction model for MOM}

To determine independent risk factors for MOM, we firstly conducted a univariate analysis by taking clinicopathological parameters and SHR expression status into account. This showed that age less than 50 years, premenopausal status, the presence of vessel and/or lymphatic thrombus, low differentiation, more advanced $\mathrm{pT}$ stage (depth of tumor invasion), more advanced $\mathrm{pN}$ stage (number of the positive lymph node), more advanced AJCC pathological stage, and 
Table 4 Univariate Cox regression analysis of clinicopathological variables of female GC patients with MOM

\begin{tabular}{|c|c|c|c|}
\hline Variables & HR & $95 \% \mathrm{Cl}$ & $P$ value \\
\hline Menstruation status (premenopausal) & 6.02 & $3.23-11.21$ & $<0.001$ \\
\hline Vessel/lymphatic thrombus (positive thrombus) & 22.19 & $6.91-71.29$ & $<0.001$ \\
\hline Differentiation (low differentiation) & 16.36 & $2.35-114.07$ & 0.005 \\
\hline Number of positive lymph nodes (more advanced pN stage) & 3.01 & $2.27-4.00$ & $<0.001$ \\
\hline AJCC TNM stage (more advanced stage) & 35.68 & 13.69-93.01 & $<0.001$ \\
\hline Expression status of ER $\alpha$ (positive ER $\alpha$ expression) & 0.94 & $0.34-2.61$ & 0.910 \\
\hline Expression status of ER $\beta$ (positive ER $\beta$ expression) & 0.24 & $0.12-0.52$ & $<0.001$ \\
\hline
\end{tabular}

GC, gastric cancer; MOM, metachronous ovarian metastasis; AJCC, American Joint Committee on Cancer; TNM, tumor (T), nodes (N), and metastases (M); HR, hazard ratio; $\mathrm{Cl}$, confidence interval; ER $\alpha$, estrogen receptor $\alpha$; ER $\beta$, estrogen receptor $\beta$; PR, progesterone receptor.

Table 5 Stepwise Cox regression model-based multivariate analysis of clinicopathological variables of female GC patients with MOM

\begin{tabular}{llc}
\hline Variables & HR & $95 \%$ Cl \\
\hline Menstruation status (premenopausal) & 3.15 & $1.66-5.98$ \\
Depth of tumor invasion (more advanced pT stage) & 3.79 & $2.14-6.69$ \\
Number of positive lymph nodes (more advanced pN stage) & 1.85 & $1.35-2.54$ \\
Expression status of ER $\beta$ (positive ER $\beta$ expression) & 0.33 & $0.15-0.7$ \\
\hline
\end{tabular}

GC, Gastric cancer; MOM, Metachronous ovarian metastasis; HR, hazard ratio; Cl, confidence interval; ER $\beta$, estrogen receptor $\beta$.

negative expression of ER $\beta$ were all significantly associated with an increased risk of MOM $(\mathrm{P}<0.05)$. In contrast, the expression status of $\mathrm{ER} \alpha$ and PR were statistically irrelevant for ovarian relapse after initial gastrectomy (Table 4).

We then conducted a Cox proportional hazards regression model-based multivariate analysis and determined that premenopausal status [hazardous ratio (HR) 3.15; 95\% CI, 1.66-5.98], more advanced pT stage (HR $3.79,95 \%$ CI, 2.14-6.69), more advanced pN stage (HR $1.85,95 \%$ CI, 1.35-2.54), and positive expression of ER $\beta$ (HR 0.33, 95\% CI, 0.15-0.7) were four independent factors predicting the occurrence of MOM $(\mathrm{P}<0.01)$ (Table 5).

Furthermore, to facilitate the timely detection of operated patients at high risk of developing MOM, we established a risk prediction model in PHS format by adopting and modifying the polygenic risk score (PRS) and PHS as previously described $(15,16)$. The calculation of PHS was based on the four independent factors and their corresponding HR values (see equation below).

Polyfactorial Hazard Score $(P H S)=\sum_{i}^{n} H R_{i} \times$ independent factor $_{i} \quad[1]$

As the risk of ovarian relapse is proportional to PHS, we then determined the best cutoff value to discriminate between patients at high and low risk of ovarian relapse, and the probability is manifest in Figure $3(\mathrm{P}<0.0001)$. Further, the 1-, 2-, and 3-year occurrence rates of MOM in highrisk patients were estimated at $17.8 \%, 33.7 \%$, and $46.2 \%$, respectively.

\section{Discussion}

Ovarian metastasis from GC, also termed as Krukenberg tumor, was first reported by the German physician Friedrich Ernst Krukenberg in 1896. Metastatic ovarian tumors arise from multiple primary sites, including the gastrointestinal tract, biliary tract, breast, and even 

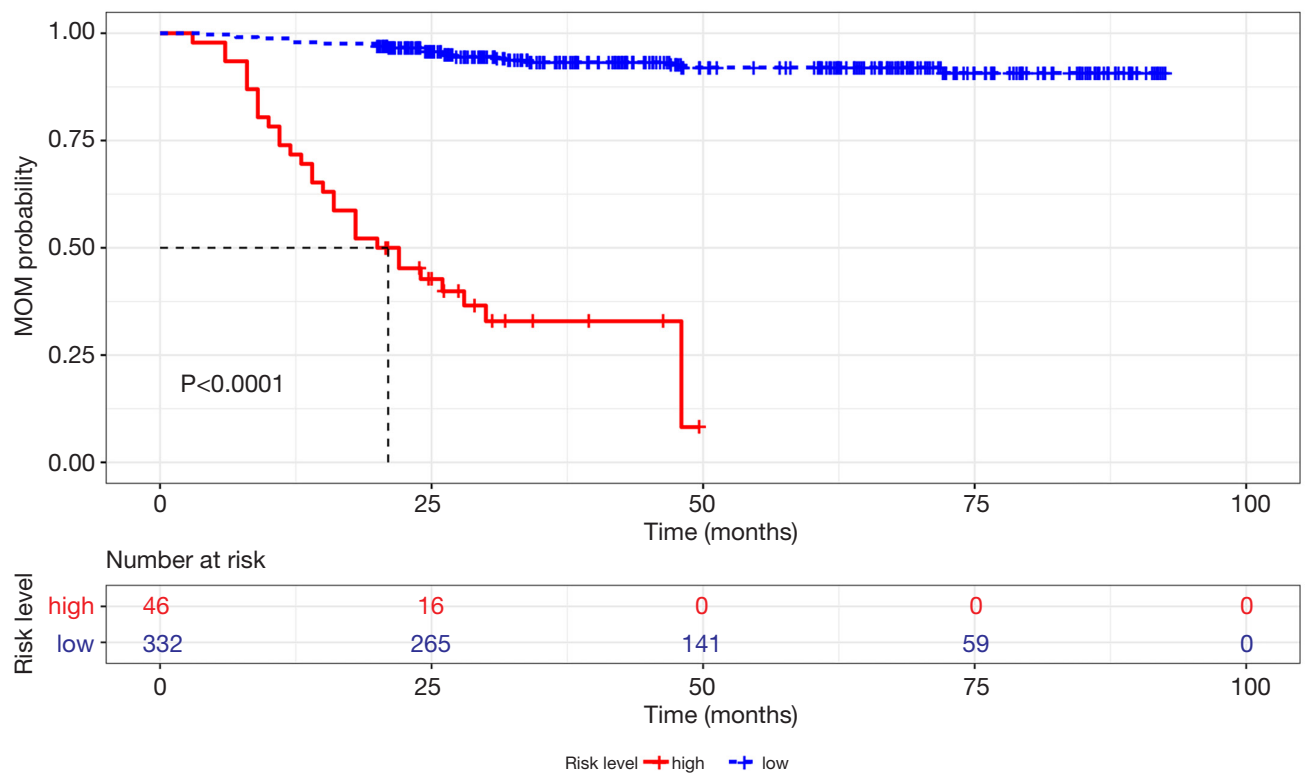

Figure 3 Kaplan-Meier ovarian relapse-free curve demonstrates the rate of MOM of high and low-risk groups partitioned by the best cutoff value. MOM, metachronous ovarian metastasis.

urinary bladder, although the stomach remains the most frequent site of primary tumors (17). Approximately 7\% of female GC patients undergoing curative operation develop ovarian reoccurrence, which is termed MOM, and the clinicopathological parameters of these patients have been systematically reviewed in several studies. The most extensive study to date, conducted by Feng et al., reviewed 63 cases of ovarian metastasis after gastrectomy and showed that the mean age of initial diagnosis of GC was 45 years, $65.1 \%$ of patients were premenopausal, and the mean interval between ovarian metastasis and primary GC was 16 months. Most primary tumors were reportedly at the T4 pathological stage (87.3\%) and N2-3 (68.3\%), 85.7\% of metastatic ovary foci were bilateral, and $73 \%$ of cases were positive for peritoneal membrane seeding, leading to an R0 resection rate as low as $31.7 \%$. Similar findings were demonstrated by other study groups $(5,8,18)$, and data from our investigation generally conforms with these results (see Results section).

The pathogenesis of ovarian metastasis from GC remains undetermined. The classic model of direct tumor seeding across the peritoneal and pelvic cavity has been gradually replaced by recognizing that multiple factors contribute to ovarian metastasis (19). Notably, retrograde lymphatic spread is increasingly recognized as the major route of ovarian metastasis considering the richness of lymphatic tissues in the stomach-ovary axis (12). Malignant cells in the lymphatic plexus of the gastric mucosa and submucosa spread through retroperitoneal lymph nodes and converge with ovarian reticular lymphatic reflux in the waist lymph nodes so that the interlinked lymphatic vessels facilitate the paradoxical metastasis of malignant cells to the ovary $(13,20)$. This is in line with our finding that the occurrence of ovarian metastasis is significantly correlated with the number of positive lymph nodes and is also supported by the previously reported observation that most metastatic foci in the ovary occur in areas of rich lymphatic tissue such as the hilum and cortex, rather than on the surface with where there is little lymphatic content (17). Hematogenous metastasis is also recognized as a significant route of ovarian metastasis as it frequently occurs in premenopausal patients whose ovaries are in a status of greater vascularity (21). Additionally, the observation that metastasis overwhelmingly occurs in bilateral ovaries instead of unilaterally also supports the pattern that cancer cells are spread via a vascular route $(5,7)$.

The prognosis of GC patients developing MOM is remarkably poor, with the median overall survival reportedly ranging from 11 to 21.7 months $(5,7,11,18)$. To address this challenge, the early detection of ovarian reoccurrence is urgently needed to provide timely medical intervention $(22,23)$. Unfortunately, this remains a major 
challenge, as most patients with ovarian reoccurrence are either asymptomatic or demonstrate non-specific symptoms such as lower abdominal discomfort, irregular bleeding, and weight loss (19). While it is essential to identify independent risk factors for the occurrence of MOM and establish a risk prediction model accordingly, most previous studies have focused on determining prognostic factors for patients with ovarian reoccurrence rather than identifying specific factors in patients who have undergone surgery and are at high risk of ovarian relapse. However, one study by Kim et al. in 1999 reported on independent risk factors for ovarian reoccurrence based on a systematic revision of the clinicopathological parameters of 690 female GC patients undergoing curative gastrectomy (8). In that study, factors including age, menopausal status, histologic classification, Lauren classification, greatest tumor dimension, tumor size, depth of invasion, number of positive lymph node, and AJCC staging were compared between 32 patients with MOM and 658 patients without, and the number of positive lymph nodes and age were reported as two independent risk factors for the occurrence of ovarian relapse. Furthermore, a risk prediction model which partitioned patients into high-risk (positive lymph nodes $>6$ and age $<50$ years), intermediate-risk (positive lymph nodes $>6$ and age $\geq 50$ years) and low-risk groups (positive lymph nodes $\leq 6$ and age $<>\geq 50$ years) showed an estimated 3 -year ovarian relapse rate of $39.5 \%$ (95\% CI, 23.8-55.7\%), 10.7\% (95\% CI, $0.2-21.1 \%$ ), and $2.1 \%$ (95\% CI, $0.5-3.8 \%$ ), respectively. However, in our analysis, premenopausal patients and those with more advanced pathological $\mathrm{T}$ stage and $\mathrm{N}$ stages were significantly associated with an increased risk of ovarian relapse. It is worth noting that menstrual status was not identified as an independent risk factor in the study by Kim et al. as their record of menstrual status was incomplete, despite their compensation by using the average Korean women menopausal age (50 years) as a cutoff value. By contrast, our complete record of menstrual status enabled us to incorporate this factor into multivariate analysis in which premenopause outcompeted age as an independent risk factor.

Another independent risk factor for ovarian recurrence identified in our study was the negative expression of ER $\beta$. This finding highlighted the significance of estrogen and its receptors in GC progression and its ovarian metastasis, especially considering the impact of elevated sex hormone activity on the reproductive organs, including the ovaries, in young females $(24,25)$. Estrogen exerts a broad influence on multiple aspects of malignant cells, the process of which is mainly mediated by its receptors such as ER $\alpha$ and ER $\beta$ (26). Once activated by estrogen, ER translocates into the nucleus and function as nuclear transcription factors that influence the transcription activity of target oncogenic or tumor-suppressing genes directly and/or indirectly binding to their regulation domains (27). ERs can induce transcription by binding to a cognate DNA binding element called estrogen response elements (ERE) within the promoters and/or enhancers of target genes with a significant impact on cell growth and differentiation in breast and prostate cancer $(28,29)$, and it is increasingly recognized that ER $\beta$ mainly functions as a tumor suppressor which could potentially serve as a treatment target in cancer therapy (30). The role of ER $\beta$ in GC progression has been broadly investigated in previous studies, including Ryu et al., who profiled its expression status in 148 GC patients and found ER $\beta$ was more highly expressed in the older age group, pT1/2 stage tumor group, and Lauren's intestinal type group than in the younger age, T3/4 stage tumor and Lauren's diffuse type group. More importantly, those authors found that the negative expression of $E R \beta$ was significantly associated with a higher rate of GC recurrence and a worse 3-year overall survival rate (9). Their conclusions were also in line with other studies, which suggested the negative expression of ER $\beta$ was correlated with an unfavorable prognosis in GC patients (31-33). On the other hand, the significance of SHR expression in ovarian metastasis of patients with GC was also investigated. Yan et al. reviewed the clinicopathological data and expression status of $\mathrm{ER} \alpha, \mathrm{Er} \beta$, and PR in 103 GC patients with synchronous ovarian metastasis (SOM) undergoing treatment in a single cancer center. They found that the negative expression of ER $\beta$ and PR were both favorable prognostic factors for overall survival (34). They further conducted a similar study by reviewing the same parameters in 152 patients ( 93 patients with SOM and 59 patients with $\mathrm{MOM}$ ), and the results also indicated that the negative expression of ER $\beta$ (HR 0.404; 95\% CI, 0.2510.648; $\mathrm{P}<0.001)$ and PR (HR 0.496; 95\% CI, 0.301-0.817; $\mathrm{P}<0.001)$ unfavorably predicted the long-term survival of GC patients with ovarian metastasis (11). Unfortunately, although numerous studies have been conducted to investigate the prognostic value of SHR expression in GC patients with ovarian metastasis, the potential predictive value of SHR expression in the occurrence of MOM has not yet been specifically addressed, and our investigation is so far the only known study addressing this issue. We compared the clinicopathological features and SHR 
expression between operated GC patients with and without ovarian relapse. We conducted a Cox regression modelbased univariate and multivariate analysis, which identified negative expression of $E R \beta$ as an independent risk factor for the reoccurrence of ovarian metastasis. Our study confirmed the correlation between ER $\beta$ expression and the risk of ovarian relapse for GC patients undergoing curative surgery for the first time.

To further facilitate the early detection of ovarian relapse, we established a risk prediction model based on four independent risk factors to identify patients at high risk of developing MOM. Here, we referred to the PRS model, which initially predicted the genetic predisposition for disease based on associated variants such as single nucleotide polymorphisms (SNPs) identified by genomewide association studies (GWAS) (15). This model essentially aggregates the impact of genetic variants into a specific value that proportionally assesses the risk for a given disease such as cancer $(16,35)$. In our risk prediction model, we proposed the PHS by adopting the essence of PRS with modification. We replaced genetic variants with four independent risk factors, which were given weightings according to their hazardous ratio (HR) calculated by multivariate analysis. With the application of PHS, we were able to estimate the 1-, 2-, and 3-year occurrence rate of MOM for operated GC patients. As PHS partially enables the quantification of the risk of ovarian reoccurrence, we could better determine which group of operated patients are prone to develop MOM.

Caution must be exercised when interpreting the independent risk factors and risk prediction model as certain limitations should be considered. Since the incidence of operated GC patients developing MOM remains low, the retrospective nature and relatively low patient numbers in this study could compromise our analyses' quality (36). Furthermore, a small proportion of patients $(10.9 \%, 6 / 55)$ developed MOM over 41 months (median follow-up period in our study) after gastrectomy. Nevertheless, given that the median time to ovarian relapse was 20 months after the initial diagnosis of GC, our median follow-up time is conceivably sufficient to perform statistical analysis to identify risk factors for the reoccurrence of ovarian metastasis. Additionally, although the expression status of SHR was included in our analysis, the total number of candidate risk factors for ovarian relapse is still minimal, especially considering that our risk prediction model originated from PRS, which is based on a large number of independent risk variants by remarkably informative GWAS (37).

It is noted in our study that metastatic foci either remained confined to the ovary $(56.4 \%, 31 / 55)$ or disseminated into the pelvis $(12.7 \%, 7 / 55)$ and abdominal peritoneum $(30.9 \%, 17 / 55)$. Previous studies demonstrated that both the treatment strategy and prognosis essentially varied between patients with localized and disseminated metastatic foci. Feng et al. reported the highest incidence of peritoneal seeding as $73.0 \%$ (46/63) in their systematic revision of patients with MOM and claimed that treatment targeting peritoneal seeding-induced metastasis could improve the prognosis (7). Cheong et al. reviewed 34 cases of post-surgical ovarian metastasis and reported that metastatic tumors were either limited to the ovary $(26.5 \%, 9 / 34)$ and pelvic cavity $(23.5 \%, 8 / 34)$ or beyond $(50.0 \%, 17 / 34)$. There was also a significant difference in both median overall survival and progression-free survival between 17 patients receiving total metastasectomy (R0) and 17 patients receiving palliative metastasectomy (R1) (18). Similar results were reported by other groups claiming that patients with confined metastatic foci (ranging from $50.0 \%$ to $74.6 \%$ ) undergoing $\mathrm{R} 0$ resection were rendered a significantly prolonged survival benefit compared with patients undergoing palliative operations $(5,11)$. In our study, the number of elevated CA724 of patients with disseminated foci was borderline significantly higher than patients in the confined foci group $(\mathrm{P}=0.05)$, indicating that operated patients with ovarian reoccurrence showing abnormally elevated CA724 are more likely to have disseminated metastatic foci, which further indicates R0 resection may not be achievable and a poor prognosis is likely.

In summary, our retrospective study determined premenopausal status, depth of tumor invasion (pT stage), number of positive lymph nodes ( $\mathrm{pN}$ stage), and negative expression of ER $\beta$ as four independent risk factors for $\mathrm{MOM}$ in female $\mathrm{GC}$ patients undergoing curative gastrectomy. Based on these findings, we further established a risk prediction model which could be applied for the early detection of post-surgical ovarian relapse so that medical intervention could be provided promptly. Future investigations should introduce high throughput technology-based methods (such as genome and/or transcriptome sequencing) to identify more specific factors essentially involved in MOM's process to optimize the risk prediction model in the light of precision completeness. Such sequencing-based technologies could also identify candidate molecular signatures distinguishing patients 
with confined and disseminated metastatic foci who will be treated with distinctive strategies.

\section{Acknowledgments}

We thank the Shanghai Institute of Digestive Surgery in Ruijin hospital, affiliated with the Shanghai Jiaotong University School of Medicine and the tissue bank of the Pathology Department of Fudan University Shanghai Cancer Center, for their generous help.

Funding: This work was supported by the grant from Scientific Funding for Youth in Fudan University Shanghai Cancer Center (Project No. YJQN202016), National Nature Science Foundation (No. 81802361) and Shanghai Anticancer Association Eyas Project (Project No. 499 SACA-CY1C04).

\section{Footnote}

Reporting Checklist: The authors have completed the REMARK reporting checklist. Available at http://dx.doi. org/10.21037/atm-21-1419

Data Sharing Statement: Available at http://dx.doi. org/10.21037/atm-21-1419

Peer Review File: Available at http://dx.doi.org/10.21037/ atm-21-1419

Conflicts of Interest: All authors have completed the ICMJE uniform disclosure form (available at http://dx.doi. org/10.21037/atm-21-1419). The authors have no conflicts of interest to declare.

Ethical Statement: The authors are accountable for all aspects of the work in ensuring that questions related to the accuracy or integrity of any part of the work are appropriately investigated and resolved. The study was conducted in accordance with the Declaration of Helsinki (as revised in 2013). The study was approved by the local ethics committee of the Fudan University Shanghai Cancer Center (Number: 050432-4-1911D) and Ruijin hospital affiliated to Shanghai Jiaotong University School of Medicine [Number: (2018) Linlunshen No. 151]. Informed consent was obtained from all patients.

Open Access Statement: This is an Open Access article distributed in accordance with the Creative Commons
Attribution-NonCommercial-NoDerivs 4.0 International License (CC BY-NC-ND 4.0), which permits the noncommercial replication and distribution of the article with the strict proviso that no changes or edits are made and the original work is properly cited (including links to both the formal publication through the relevant DOI and the license). See: https://creativecommons.org/licenses/by-nc-nd/4.0/.

\section{References}

1. Bray F, Ferlay J, Soerjomataram I, et al. Global cancer statistics 2018: GLOBOCAN estimates of incidence and mortality worldwide for 36 cancers in 185 countries. CA Cancer J Clin 2018;68:394-424.

2. Bernards N, Creemers GJ, Nieuwenhuijzen GA, et al. No improvement in median survival for patients with metastatic gastric cancer despite increased use of chemotherapy. Ann Oncol 2013;24:3056-60.

3. Yada-Hashimoto N, Yamamoto T, Kamiura S, et al. Metastatic ovarian tumors: a review of 64 cases. Gynecol Oncol 2003;89:314-7.

4. Zhang JJ, Cao DY, Yang JX, et al. Ovarian metastasis from nongynecologic primary sites: a retrospective analysis of 177 cases and 13-year experience. J Ovarian Res 2020;13:128.

5. Jun SY, Park JK. Metachronous ovarian metastases following resection of the primary gastric cancer. J Gastric Cancer 2011;11:31-7.

6. Wang J, Shi YK, Wu LY, et al. Prognostic factors for ovarian metastases from primary gastric cancer. Int $\mathrm{J}$ Gynecol Cancer 2008;18:825-32.

7. Feng Q, Pei W, Zheng ZX, et al. Clinicopathologic characteristics and prognostic factors of 63 gastric cancer patients with metachronous ovarian metastasis. Cancer Biol Med 2013;10:86-91.

8. Kim NK, Kim HK, Park BJ, et al. Risk factors for ovarian metastasis following curative resection of gastric adenocarcinoma. Cancer 1999;85:1490-9.

9. Ryu WS, Kim JH, Jang YJ, et al. Expression of estrogen receptors in gastric cancer and their clinical significance. J Surg Oncol 2012;106:456-61.

10. Tang W, Liu R, Yan Y, et al. Expression of estrogen receptors and androgen receptor and their clinical significance in gastric cancer. Oncotarget 2017;8:40765-77.

11. Yu P, Huang L, Cheng G, et al. Treatment strategy and prognostic factors for Krukenberg tumors of gastric origin: report of a 10-year single-center experience from China. 
Oncotarget 2017;8:82558-70.

12. Kubeček O, Laco J, Špaček J, et al. The pathogenesis, diagnosis, and management of metastatic tumors to the ovary: a comprehensive review. Clin Exp Metastasis 2017;34:295-307.

13. Al-Agha OM, Nicastri AD. An in-depth look at Krukenberg tumor: an overview. Arch Pathol Lab Med 2006;130:1725-30.

14. Gan L, He J, Zhang X, et al. Expression profile and prognostic role of sex hormone receptors in gastric cancer. BMC Cancer 2012;12:566.

15. Seibert TM, Fan CC, Wang Y, et al. Polygenic hazard score to guide screening for aggressive prostate cancer: development and validation in large scale cohorts. BMJ 2018;360:j5757.

16. Mavaddat N, Michailidou K, Dennis J, et al. Polygenic Risk Scores for Prediction of Breast Cancer and Breast Cancer Subtypes. Am J Hum Genet 2019;104:21-34.

17. Kiyokawa T, Young RH, Scully RE. Krukenberg tumors of the ovary: a clinicopathologic analysis of 120 cases with emphasis on their variable pathologic manifestations. Am J Surg Pathol 2006;30:277-99.

18. Cheong JH, Hyung WJ, Chen J, et al. Surgical management and outcome of metachronous Krukenberg tumors from gastric cancer. J Surg Oncol 2004;87:39-45.

19. Agnes A, Biondi A, Ricci R, et al. Krukenberg tumors: Seed, route and soil. Surg Oncol 2017;26:438-45.

20. Kakushima N, Kamoshida T, Hirai S, et al. Early gastric cancer with Krukenberg tumor and review of cases of intramucosal gastric cancers with Krukenberg tumor. J Gastroenterol 2003;38:1176-80.

21. Yook JH, Oh ST, Kim BS. Clinical prognostic factors for ovarian metastasis in women with gastric cancer. Hepatogastroenterology 2007;54:955-9.

22. Aurello P, Berardi G, Antolino L, et al. Is a Surgical Approach Justified in Metachronous Krukenberg Tumor from Gastric Cancer? A Systematic Review. Oncol Res Treat 2018;41:644-9.

23. Ma F, Li Y, Li W, et al. Metastasectomy Improves the Survival of Gastric Cancer Patients with Krukenberg Tumors: A Retrospective Analysis of 182 patients. Cancer Manag Res 2019;11:10573-80.

24. Sullivan SD, Sarrel PM, Nelson LM. Hormone replacement therapy in young women with primary ovarian insufficiency and early menopause. Fertil Steril 2016;106:1588-99.

25. Folkerd EJ, Dowsett M. Influence of Sex Hormones on Cancer Progression. Journal of Clinical Oncology
2010;28:4038-44.

26. Gallo D, De Stefano I, Grazia Prisco M, et al. Estrogen receptor beta in cancer: an attractive target for therapy. Curr Pharm Des 2012;18:2734-57.

27. Ikeda K, Horie-Inoue K, Inoue S. Identification of estrogen-responsive genes based on the DNA binding properties of estrogen receptors using high-throughput sequencing technology. Acta Pharmacol Sin 2015;36:24-31.

28. Katzenellenbogen BS, Katzenellenbogen JA. Estrogen receptor transcription and transactivation: Estrogen receptor alpha and estrogen receptor beta: regulation by selective estrogen receptor modulators and importance in breast cancer. Breast Cancer Res 2000;2:335-44.

29. Omoto $\mathrm{Y}$, Iwase H. Clinical significance of estrogen receptor $\beta$ in breast and prostate cancer from biological aspects. Cancer Sci 2015;106:337-43.

30. Mal R, Magner A, David J, et al. Estrogen Receptor Beta (ER $\beta)$ : A Ligand Activated Tumor Suppressor. Front Oncol 2020;10:587386.

31. Xu CY, Guo JL, Jiang ZN, et al. Prognostic role of estrogen receptor alpha and estrogen receptor beta in gastric cancer. Ann Surg Oncol 2010;17:2503-9.

32. Ur Rahman MS, Cao J. Estrogen receptors in gastric cancer: Advances and perspectives. World J Gastroenterol 2016;22:2475-82.

33. Jang Y, Mok Y. The estrogenic hormone effect in gastric cancer. Journal of Clinical Oncology 2018;36:85.

34. Yan D, Du Y, Dai G, et al. Management Of Synchronous Krukenberg Tumors From Gastric Cancer: a Single-center Experience. J Cancer 2018;9:4197-203.

35. Lambert SA, Abraham G, Inouye M. Towards clinical utility of polygenic risk scores. Hum Mol Genet 2019;28:R133-42.

36. Mann CJ. Observational research methods. Research design II: cohort, cross sectional, and case-control studies. Emerg Med J 2003;20:54-60.

37. Lewis CM, Vassos E. Polygenic risk scores: from research tools to clinical instruments. Genome Med 2020;12:44.

(English Language Editors: B. Draper and J. Chapnick)

Cite this article as: Gao J, Weng W, Qu X, Huang B, Zhang Y, Zhu Z. Risk factors predicting the occurrence of metachronous ovarian metastasis of gastric cancer. Ann Transl Med 2021;9(13):1049. doi: 10.21037/atm-21-1419 


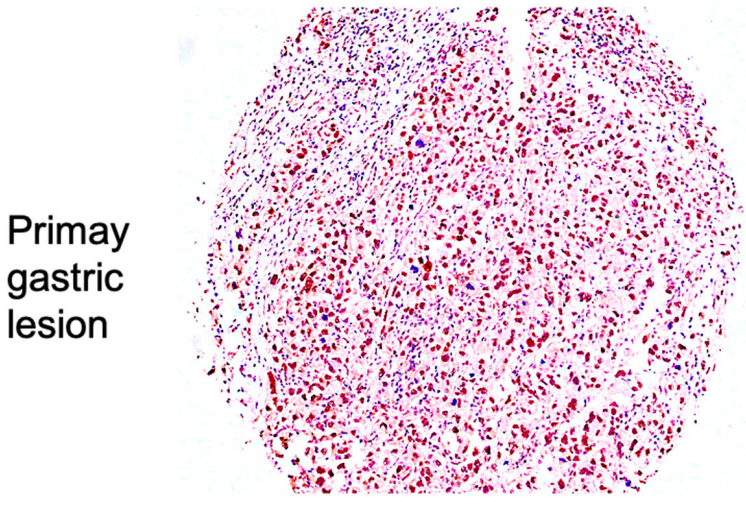

$10 \times$

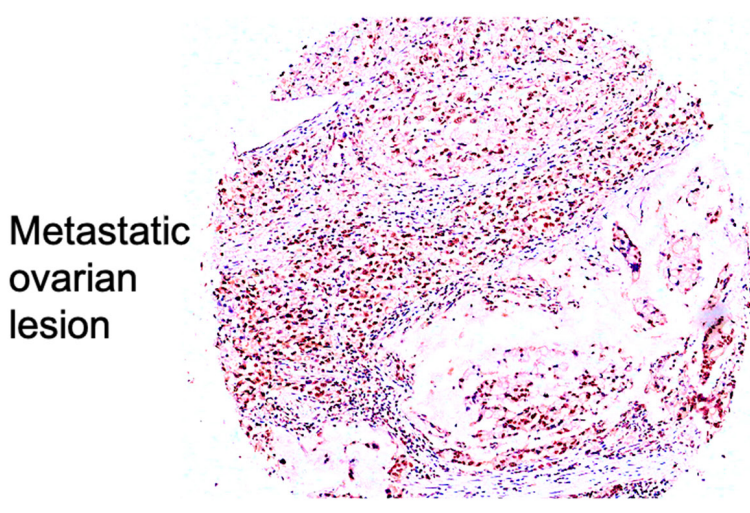

$10 \times$

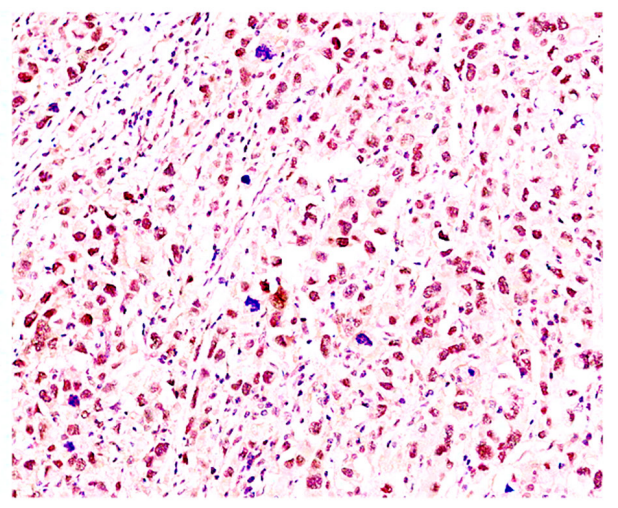

$20 \times$

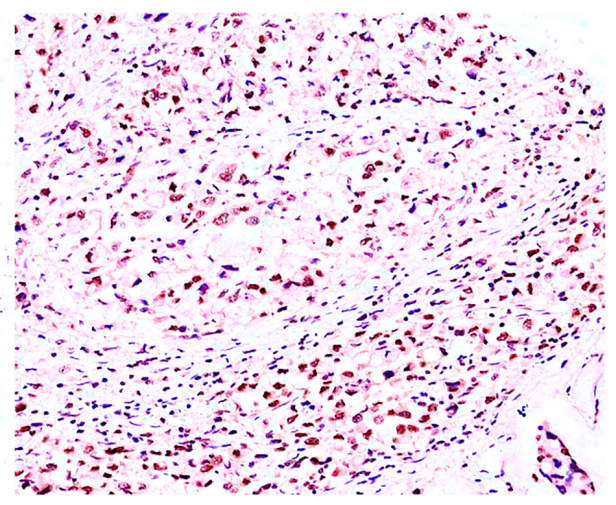

$20 x$

Figure S1 Representative immunohistochemical staining of $\operatorname{ER} \beta$ on paired tumors (primary gastric tumor $v s$. metastatic ovarian tumor) of operated GC patients with MOM. Er $\beta$, estrogen receptor $\beta$; GC, gastric cancer; MOM, metachronous ovarian metastasis. 